\title{
Interacting Electrons in Graphene: Fermi Velocity Renormalization and Optical Response
}

\author{
T. Stauber, ${ }^{1}$ P. Parida, ${ }^{2}$ M. Trushin, ${ }^{3}$ M. V. Ulybyshev, ${ }^{2}$ D. L. Boyda, ${ }^{4,5}$ and J. Schliemann ${ }^{2}$ \\ ${ }^{1}$ Departamento de Teoría y Simulación de Materiales, Instituto de Ciencia de Materiales de Madrid, CSIC, E-28049 Madrid, Spain \\ ${ }^{2}$ Institute for Theoretical Physics, University of Regensburg, D-93040 Regensburg, Germany \\ ${ }^{3}$ Department of Physics, University of Konstanz, D-78457 Konstanz, Germany \\ ${ }^{4}$ Far Eastern Federal University, Sukhanova 8, Vladivostok 690950, Russia \\ ${ }^{5}$ ITEP, B. Cheremushkinskaya 25, Moscow 117218, Russia
}

(Received 12 April 2017; published 27 June 2017)

\begin{abstract}
We have developed a Hartree-Fock theory for electrons on a honeycomb lattice aiming to solve a longstanding problem of the Fermi velocity renormalization in graphene. Our model employs no fitting parameters (like an unknown band cutoff) but relies on a topological invariant (crystal structure function) that makes the Hartree-Fock sublattice spinor independent of the electron-electron interaction. Agreement with the experimental data is obtained assuming static self-screening including local field effects. As an application of the model, we derive an explicit expression for the optical conductivity and discuss the renormalization of the Drude weight. The optical conductivity is also obtained via precise quantum Monte Carlo calculations which compares well to our mean-field approach.
\end{abstract}

DOI: 10.1103/PhysRevLett.118.266801

Introduction.-The role of Coulomb interactions in graphene is still an open and important question [1], also in view of the regime of hydrodynamic electron liquids in which the electron-electron interaction represents the dominant scattering process [2,3]. The influence becomes especially crucial around the neutrality point, and it has been manifested through the measurement of the effective cyclotron mass [4], by scanning tunneling spectroscopy $[5,6]$, by direct ARPES of the Dirac cones [7], by quantum capacitance measurements [8], and also by Landau level spectroscopy [9], that there is a Fermi velocity renormalization when lowering the electronic density close to half-filling.

A one-loop renormalization group (RG) and analogous Hartree-Fock (HF) analysis based on the continuous Dirac model predicts the following scaling behavior $[4,10]$ :

$$
\frac{v_{F}^{*}}{v_{F}}=1+\frac{\alpha}{4} \ln \frac{\Lambda}{k},
$$

where $\quad \alpha=\left(1 / 4 \pi \epsilon_{0} \epsilon\right)\left(e^{2} / \hbar v_{F}\right) \approx 2.2 / \epsilon$ is the finestructure constant of graphene with the bare Fermi velocity $v_{F}, \epsilon$ characterizes the static dielectric environment, and $\Lambda$ is the momentum cutoff. Moreover, $v_{F}$ denotes the bare Fermi velocity and $v_{F}^{*}$ the renormalized Fermi velocity at wave number $k$.

Equation (1) has been extended by several authors [11-15], but a recent multiloop expansion claims that perturbation theory may be inadequate particularly for suspended graphene [16]. Nonetheless, within a nonperturbative functional renormalization group analysis, the perturbative series can be summed up to again yield Eq. (1) with almost the same prefactor $\alpha / 4$ as obtained from the HF approach [17,18]. This suggests that a self-consistent mean-field theory will contain all the necessary ingredients to address interaction effects even close to the neutrality point.

The experimental data for the velocity renormalization can be fitted to Eq. (1) by adjusting the band cutoff $\Lambda$ as well as the effective dielectric screening constant $\epsilon$ [4]. Nevertheless, some ambiguities inherent to the renormalization group approach can only be resolved by resorting to a realistic tight-binding Hamiltonian rather than working with an effective low-energy theory [19]. This especially holds for the optical conductivity that has been the subject of persistent discussion regarding the constant $C$ in the expansion $\sigma^{*} / \sigma_{0}=1+C \alpha^{*}+O\left(\alpha^{* 2}\right)$, with $\sigma_{0}=e^{2} / 4 \hbar$ the universal conductivity and $\alpha^{*} / \alpha=v_{F} / v_{F}^{*}$ [14,20-27]. After 10 years of debate, one way to resolve this controversy could be an alternative, but well-defined numerical approach which still allows for analytical insight. We have therefore performed detailed HF calculations on the honeycomb lattice that hopefully will be able to shed some light on this issue from a different angle. We complement this with state-of-the-art quantum Monte Carlo calculations which now precisely determine the optical conductivity at energies of the order of the hopping parameter.

In contrast to earlier HF calculations preformed for a graphene quantum dot [28], the Dirac model [29], multilayer graphene [30], and graphene on a lattice [31] we now take into account self-screening and finite electronic densities which are shown to be crucial to explain the experimental data without the need for a fitting parameter. We further take advantage of the fact that the HF wave function is independent of the interaction strength even for a lattice model and relate this to a topological invariant which protects the 
chirality of the Dirac fermions around the nodal points. This reduces the numerical cost and further results in $\mathrm{HF}$ equations that in some limits are identical to the ones obtained from RG equations [32] and Hubbard-Stratonovich transformation [33]. The knowledge of the HF wave function further enables us to derive analytical expressions for the optical conductivity in the unscreened case with $C=1 / 4$, close to the value of Ref. [22]. Including selfscreening, we obtain $C \approx 0.05$ for suspended samples in agreement with our Monte Carlo calculations.

The Drude weight renormalization is another motivation of this work, because studies of the electromagnetic response of various classes of correlated electron materials are often based on the $f$-sum rule [34]. Integrating the optical conductivity over the spectral range is then related to the Drude weight $D$, which is independent of the interaction in a Galilean invariant system. However, this is not the case for Dirac systems anymore, and electron-electron interactions modify the Drude weight in a nontrivial way that is larger than the Drude weight of the noninteracting system $[19,35]$. Sum rule analysis in Dirac systems [36,37] have thus to be taken with care. Renormalization of the Drude weight is also of interest for plasmonics in Dirac systems as the plasmon energy scales as $\sqrt{D}$ [38]. Within our approach, we can analytically discuss the Drude weight for electronic densities close to half-filling.

The model.-We will model interacting Dirac fermions in graphene within a nearest-neighbor tight-binding model using HF theory. An important insight of this work is that the crystallographic structure factor is a topological invariant that does not depend on the ground state as long as it obeys the threefold symmetry of the underlying honeycomb lattice; see the Supplemental Material [39], which also includes Refs. [40-48]. If we do not allow for chiral [49] or time-reversal [50] symmetry breaking, the HF wave function of the interacting system is then given by the noninteracting wave function. This is reminiscent of the absence of wave function renormalization in the $\mathrm{RG}$ approach [10].

In order to consistently include electron-electron interactions, the Coulomb potential needs to be periodic as discussed by Jung and MacDonald [31]. This introduces screening of the Coulomb interaction at small distances. If one further incorporates screening at large distances due to tight-binding electrons, the interaction potential is not translationally invariant anymore and additional nondiagonal local field effects need to be considered, see Supplemental Material [39]. To calculate the atomic orbital form factor $f(\mathbf{q})=\int d \mathbf{r} e^{-i \mathbf{q} \cdot \mathbf{r}}|\zeta(\mathbf{r})|^{2}$, with $\zeta(\mathbf{r})$ being the one-electron atomic wave function, we take the full angular dependence of the wave function into account in contrary to Refs. [31] or [26].

Hartree-Fock theory.-As mentioned above, the relative phase between the spinor components is a topological invariant and the single particle HF Hamiltonian $H_{\mathbf{k}}$ for any interaction strength can be written as

$$
H_{\mathbf{k}}=-\mathcal{E}_{\mathbf{k}}\left[\cos \left(\varphi_{\mathbf{k}}\right) \sigma_{x}-\sin \left(\varphi_{\mathbf{k}}\right) \sigma_{y}\right],
$$

where $e^{i \varphi_{\mathbf{k}}}=\phi_{\mathbf{k}} /\left|\phi_{\mathbf{k}}\right|$ with the crystallographic structure factor $\phi_{\mathbf{k}}=\sum_{i} e^{i \mathbf{k} \cdot \delta_{i}}$, and the three nearest-neighbor lattice vectors $\delta_{i}, i=1,2,3$. In the Supplemental Material [39], we present the mean-field theory of a more general Hamiltonian which also includes a momentum dependent mass and energy shift for the sake of generality [39].

The above Hamiltonian is characterized by the renormalized energy dispersion $\mathcal{E}_{\mathbf{k}}$, which is determined selfconsistently by the following equation:

$$
\mathcal{E}_{\mathbf{k}}=\mathcal{E}_{\mathbf{k}}^{0}+\frac{1}{2 A} \sum_{\mathbf{k}^{\prime} \in 1 . \mathrm{BZ}} U\left(\mathbf{k}-\mathbf{k}^{\prime}\right) e^{i\left(\varphi_{\mathbf{k}^{\prime}}-\varphi_{\mathbf{k}}\right)} F_{\mathbf{k}^{\prime}},
$$

where we introduced $\mathcal{E}_{\mathbf{k}}^{0}=t\left|\phi_{\mathbf{k}}\right|$ as the noninteracting dispersion relation, $t$ is the tunnel-matrix element between the nearest carbon atoms, and $A$ denotes the sample area. Further, we have $F_{\mathbf{k}}=n_{F}\left(-\mathcal{E}_{\mathbf{k}}\right)-n_{F}\left(\mathcal{E}_{\mathbf{k}}\right)$ with the Fermi distribution function $n_{F}(\epsilon)=\left(e^{\beta(\epsilon-\mu)}+1\right)^{-1}$ and the chemical potential $\mu$ at a finite temperature $\beta=1 /\left(k_{B} T\right)$. The Coulomb potential conserving the lattice symmetry and including the local field effects reads

$$
\begin{aligned}
U(\mathbf{q})= & \sum_{\mathbf{G}, \mathbf{G}^{\prime}} e^{-i \mathbf{G}^{\prime} a} f^{*}(\mathbf{q}+\mathbf{G}) f\left(\mathbf{q}+\mathbf{G}^{\prime}\right) \\
& \times\left[\delta_{\mathbf{G}, \mathbf{G}^{\prime}}-v_{\mathbf{G}}(\mathbf{q}) \chi_{\mathbf{G}, \mathbf{G}^{\prime}}(\mathbf{q})\right]^{-1} v_{\mathbf{G}^{\prime}}(\mathbf{q}),
\end{aligned}
$$

where $\mathbf{G}, \mathbf{G}^{\prime}$ are the reciprocal lattice vectors, $f(\mathbf{q})$ is the form factor, $v_{\mathbf{G}}(\mathbf{q})=\left(e^{2} / 2 \epsilon_{0} \epsilon\right)(1 /|\mathbf{q}+\mathbf{G}|)$ is the Fouriertransformed screened Coulomb potential, and $\chi_{\mathbf{G}, \mathbf{G}^{\prime}}(\mathbf{q})$ is the static polarizability matrix with local field effects; see the Supplemental Material [39]. Neglecting the self-consistency by replacing $\mathcal{E}_{\mathbf{k}} \rightarrow \mathcal{E}_{\mathbf{k}}^{0}$ on the right-hand side of Eq. (3), we arrive at the same equation that was obtained from a Hubbard-Stratonovich transformation on the lattice [33].

In Fig. 1, the renormalized band structure of neutral graphene is shown for $t=3.1 \mathrm{eV}\left(v_{F}=10^{6} \mathrm{~m} / \mathrm{s}\right)$ between the high symmetry points of the Brillouin zone for various coupling constant $\alpha$, i.e., for different dielectric environments $\epsilon$. By this, we can discuss suspended graphene $(\epsilon=1, \alpha=2.2)$, graphene on top of silicon $(\epsilon=2.45$, $\alpha=0.9), \quad$ or hBN-encapsulated graphene $(\epsilon=4.9$, $\alpha=0.45$ ). The solid lines refer to self-screened interactions which are compared to the dispersion due to bare interaction for $\alpha=2.2$ (dashed line). The inset shows the region close to the Dirac point where only slight deviations from the linear behavior can be seen.

Fermi velocity renormalization. - At half-filling $\mu=0$, $T=0$ and no self-screening, Eq. (3) is an explicit equation. This yields the analytical expression of Eq. (1) when assuming $U(\mathbf{q})=v_{\mathbf{G}=0}(\mathbf{q})$ and converting the summation over the Brillouin zone by the Dirac cone approximation. Solving Eq. (3) numerically, we obtain a fit for the cutoff parameter with $\Lambda \approx 1.75 \AA^{-1}$. This is in contrast to Ref. [31], where $\Lambda \approx 20 \AA^{-1}$ was obtained, but agrees well 


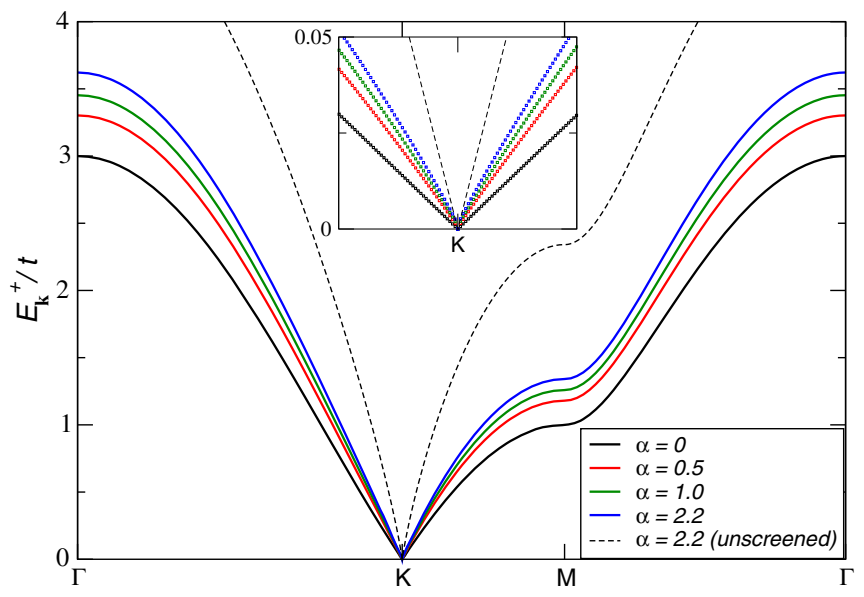

FIG. 1. Band structure (undoped) along high symmetry directions for various fine-structure constants $\alpha$ and self-screening (solid lines). For suspended graphene ( $\alpha=2.2, t=3.1 \mathrm{eV}$ ), also the unscreened dispersion is shown (dashed line). Inset: Enlargement of the dispersion around the Dirac cone.

with the usual argument of fixing $\Lambda$ by conserving the total number of states in the Brillouin zone when compared to the tight-binding model, yielding $\Lambda \approx 1.58 \AA^{-1}$. The precise value of $\Lambda$ depends only weakly on the nonuniversal shortranged Coulomb interaction; see the Supplemental Material [39].

For unscreened interaction, the correction to the Fermi velocity $v_{F}^{*} / v_{F}-1$ is proportional to $\alpha$ and the result is scale independent of the hopping parameter $t$. At zero doping, self-screening can be incorporated by $\alpha \rightarrow \alpha / \epsilon^{\mathrm{RPA}}$ because $\epsilon^{\mathrm{RPA}}=1+(\pi / 2) \alpha$ is momentum independent within the Dirac-cone approximation [51]. This yields good agreement with the experimental data of Ref. [4] for small densities $n \lesssim 20 \times 10^{10} \mathrm{~cm}^{-2}$ without the need for any fitting parameter; see the magenta curve of Fig. 2 .

For densities $n \gtrsim 20-60 \times 10^{10} \mathrm{~cm}^{-2}$, there is a decrease of the Fermi velocity that cannot be accounted for by the results for neutral graphene. Since it might be due to screening at finite densities, we incorporated the momentum dependent polarization function as outlined above. Even though the renormalized Fermi velocity now depends on $\alpha$ as well as on $t$ in a nontrivial way, in the asymptotic limit it becomes independent of $t$ for $\mu=0$.

On the left-hand side of Fig. 2, we show the solution of Eq. (3) using the bare (black squares) and the self-consistent (blue stars) polarization function. The bare solution agrees well with the experimental data for suspended graphene up to $n \lesssim 40 \times 10^{10} \mathrm{~cm}^{-2}$, but at higher densities the experimental data drop whereas the theoretical value remains approximately constant (on a logarithmic scale). This has to be contrasted with the experimental data for hBN-encapsulated graphene [8], where good agreement is obtained over the whole density range up to $n \sim 5 \times 10^{12} \mathrm{~cm}^{-2}$, see Fig. 2 (right). For the particular choice of the bare hopping amplitude in the two cases,

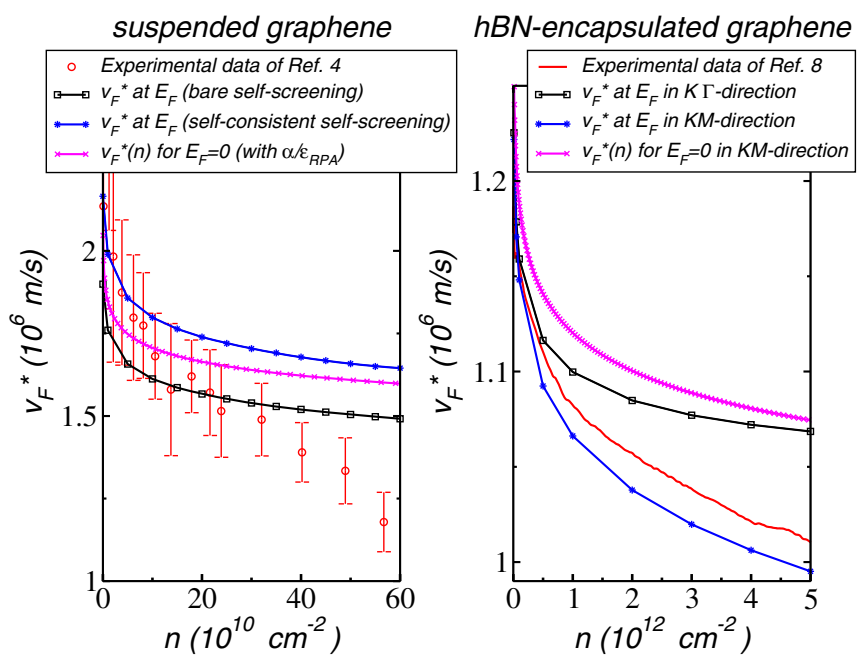

FIG. 2. The renormalized Fermi velocity $v_{F}^{*}$ for suspended $(\epsilon=1$ and $t=3.1 \mathrm{eV})$ and hBN-encapsulated $(\epsilon=4.9$ and $t=2.6 \mathrm{eV}$ ) graphene. Left-hand side for suspended graphene: The experimental data of Ref. [4] compared to $v_{F}^{*}$ at the Fermi surface based on the bare and self-consistent self-screened Coulomb interaction. Right-hand side for hBN-encapsulated graphene: The experimental data of Ref. [8] compared to $v_{F}^{*}$ at the Fermi surface in $K \Gamma$ (black squares) and $K M$ (blue stars) directions based on the bare self-screened Coulomb interaction. In both cases, the result for $v_{F}^{*}$ at the neutrality point as function of the electronic density $n$ is also shown based on the unscreened interaction with $\alpha=\alpha / \epsilon^{\mathrm{RPA}}$.

i.e., $t=3.1$ and $t=2.6 \mathrm{eV}$, respectively, we were guided by the original Refs. $[4,8]$, where similar values were used; see also Ref. [52].

Optical response.- Let us now turn to the interaction effects on the optical response, first discussed for Dirac electrons in Ref. [53] in the case of electron-phonon coupling. To do so, we will couple the gauge field via the Peierls substitution by replacing $\mathbf{k} \rightarrow \mathbf{k}+(e / \hbar) \mathbf{A}$ in the mean-field Hamiltonian $H_{\mathbf{k}}$. This procedure provides the correct vertex correction such that the optical $f$-sum rule is satisfied.

Since the HF wave function is known, the optical conductivity can be deduced from the noninteracting tight-binding model [54] by replacing the bare dispersion by the renormalized one. For momenta close to the Dirac point, the dispersion is isotropic with $v_{F}^{*} / v_{F}=$ $1+C(\alpha) \alpha \ln \Lambda / k$. We then obtain for small radiation frequencies $\omega \ll \hbar$ the following result [39]:

$$
\frac{\sigma^{*}}{\sigma_{0}}=1+C(\alpha) \alpha \frac{v_{F}}{v_{F}^{*}},
$$

where $\sigma_{0}=\left(e^{2} / 4 \hbar\right)$ denotes the universal conductivity. For unscreened interaction, we obtain explicitely $C=1 / 4$ (see Supplemental Material [39]) as mentioned in the introduction. This compares well with $C \approx 0.26$ obtained in Refs. [22,24].

For the self-screened interaction, $C \rightarrow C(\alpha)$ becomes a function with $C(\alpha \rightarrow 0) \rightarrow 0.25$ (unscreened limit) and 

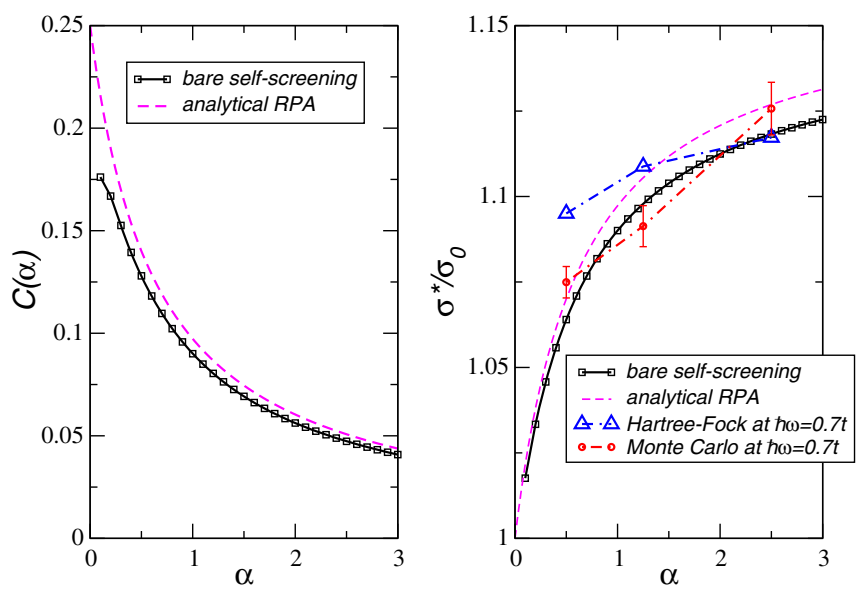

FIG. 3. Left: The correction to the optical conductivity $C(\alpha)$ of Eq. (5) compared to the result expected from the RPA, i.e., $\left[4 \epsilon^{\mathrm{RPA}}\right]^{-1}=\{4[1+(\pi / 2) \alpha]\}^{-1}$. Right: The same for the conductivity of Eq. (5) with $v_{F}^{*} / v_{F}=1$ compared to the conductivity at $\hbar \omega=0.7 t$ obtained from Hartree-Fock (blue triangle) and quantum Monte Carlo (red circle) calculations.

$C(\alpha=2.5) \approx 0.05$ for suspended graphene with $t=2.7 \mathrm{eV}$. It is interesting to note that the universal factor of the scaling law $C(\alpha)$ is independent of all considered hopping matrix elements from $t=2.6$ to $t=3.1 \mathrm{eV}$, and it compares well if self-screening is incorporated via RPA within the Dirac cone approximation, i.e., $C(\alpha)=\{4[1+(\pi / 2) \alpha]\}^{-1}$. This is shown on the left of Fig. 3; assuming a small, but finite electronic density would further decrease the constant $C(\alpha)$.

On the right-hand side, we plot the same for the conductivity of Eq. (5) with $v_{F}^{*} / v_{F}=1$. This is compared to the conductivity at $\hbar \omega=0.7 t$ as obtained from HF and quantum Monte Carlo calculations; see the Supplemental Material [39]. We obtain good agreement between the two approaches for suspended graphene which justifies our mean-field approach.

Drude weight.-In a Galilean invariant system, the Drude weight $D$ is independent of the interaction. However, this is not the case for Dirac systems and electron-electron interactions modify the Drude weight in a nontrivial way that is larger than the Drude weight of the noninteracting system $[19,35]$. Making use of the fact that the HF wave function is given by the noninteracting wave function, one can obtain an analytical expression for the Drude weight from the optical $f$-sum rule (see Supplemental Material [39]):

$$
D_{i i}=\left(\frac{e}{\hbar}\right)^{2} \frac{g_{s}}{A} \sum_{\mathbf{k} \in 1 . B Z, s= \pm} s\left[\partial_{k_{i}}^{2} \mathcal{E}_{\mathbf{k}}\right] n_{F}\left(s \mathcal{E}_{\mathbf{k}}\right)
$$

with $g_{s}=2$ the spin degeneracy, and $i=x, y$. This expression for the Drude weight in the presence of Coulomb interactions generalizes the known result for noninteracting systems: $D=e^{2} n / m$ for Schrödinger particles with the density $n$ and mass $m$ and $D=g_{s}(e / h)^{2} \pi \mu$ for for Dirac particles in graphene [38]. As might have been expected, within our mean-field theory the interacting electrons behave as independent quasiparticles with renormalized dispersion $\lambda \mathcal{E}_{\mathbf{k}}$ and we obtain [39]

$$
\frac{D^{*}}{D}=\frac{v_{F}^{*}}{v_{F}} .
$$

A similar relation was obtained in Ref. [19] in the case of unscreened interactions. Changes due to trigonal warping and finite temperature can also be discussed by our approach.

Summary.-We presented a realistic tight-binding approach to the electron band structure of graphene renormalized by the Coulomb interaction. We identified a topological invariant which leaves the HF wave function unchanged even in the presence of the Coulomb interaction and found analytical expressions for the optical conductivity as well as for the Drude weight. By this, we were able to link our findings to the measured optical conductivity which shows only little renormalization due to selfscreened interactions. Precise Monte Carlo calculations yield good agreement for suspended samples and support our mean-field approach. We also show that the Fermi velocity and Drude weight renormalization are the same according to the expectations of a mean-field theory.

Our results compare well with experiments for suspended as well as for $\mathrm{hBN}$-encapsulated graphene without invoking fitting parameters. But in the case of suspended graphene, we were not able to account for the velocity renormalization in the case of larger densities $n \gtrsim 40 \times 10^{10} \mathrm{~cm}^{-2}$. This effect cannot be explained by our tight-binding model and we expect the influence of ripples and corrugations, partially due to the applied gate, to be responsible for this effective screening of the longranged Coulomb interaction. This would also imply the absence of interaction renormalization of (high-density) plasmons in suspended graphene.

We acknowledge interesting discussions with Igor Herbut, Jeil Jung, Peter Kopietz, Vieri Mastropietro, and Anand Sharma. We also thank Kostya Novoselov for providing us with the experimental data. This work has been supported by Spain's MINECO under Grant No. FIS2014-57432-P, by the Comunidad de Madrid under Grant No. S2013/MIT-3007 MAD2D-CM, and by Germany's Deutsche Forschungsgemeinschaft (DFG) via SFB 689. The work of M. V. U. was supported by DFG Grant No. BU 2626/2-1. D. L. B. acknowledges the support by RFBR Grant No. 16-32-00362-mol-a.

[1] V. N. Kotov, B. Uchoa, V. M. Pereira, F. Guinea, and A. H. Castro Neto, Rev. Mod. Phys. 84, 1067 (2012).

[2] D. A. Bandurin, I. Torre, R. K. Kumar, M. Ben Shalom, A. Tomadin, A. Principi, G. H. Auton, E. Khestanova, 
K. S. Novoselov, I. V. Grigorieva, L. A. Ponomarenko, A. K. Geim, and M. Polini, Science 351, 1055 (2016).

[3] J. Crossno, J. K. Shi, K. Wang, X. Liu, A. Harzheim, A. Lucas, S. Sachdev, P. Kim, T. Taniguchi, K. Watanabe, T. A. Ohki, and K. C. Fong, Science 351, 1058 (2016).

[4] D. C. Elias, R. V. Gorbachev, A. S. Mayorov, S. V. Morozov, A. A. Zhukov, P. Blake, L. A. Ponomarenko, I. V. Grigorieva, K. S. Novoselov, F. Guinea, and A. K. Geim, Nat. Phys. 7, 701 (2011).

[5] G. Li, A. Luican, and E. Y. Andrei, Phys. Rev. Lett. 102, 176804 (2009).

[6] J. Chae, S. Jung, A. F. Young, C. R. Dean, L. Wang, Y. Gao, K. Watanabe, T. Taniguchi, J. Hone, K. L. Shepard, P. Kim, N. B. Zhitenev, and J. A. Stroscio, Phys. Rev. Lett. 109, 116802 (2012).

[7] D. A. Siegel, C.-H. Park, C. Hwang, J. Deslippe, A. V. Fedorov, S. G. Louie, and A. Lanzara, Proc. Natl. Acad. Sci. U.S.A. 108, 11365 (2011).

[8] G. L. Yu, R. Jalil, B. Belle, A. S. Mayorov, P. Blake, F. Schedin, S. V. Morozov, L. A. Ponomarenko, F. Chiappini, S. Wiedmann, U. Zeitler, M. I. Katsnelson, A. K. Geim, K. S. Novoselov, and D. C. Elias, Proc. Natl. Acad. Sci. U.S.A. 110, 3282 (2013).

[9] C. Faugeras, S. Berciaud, P. Leszczynski, Y. Henni, K. Nogajewski, M. Orlita, T. Taniguchi, K. Watanabe, C. Forsythe, P. Kim, R. Jalil, A. K. Geim, D. M. Basko, and M. Potemski, Phys. Rev. Lett. 114, 126804 (2015).

[10] J. González, F. Guinea, and M. Vozmediano, Nucl. Phys. B424, 595 (1994).

[11] T. Stauber, F. Guinea, and M. A. H. Vozmediano, Phys. Rev. B 71, 041406 (2005).

[12] E. G. Mishchenko, Phys. Rev. Lett. 98, 216801 (2007).

[13] D. E. Sheehy and J. Schmalian, Phys. Rev. Lett. 99, 226803 (2007).

[14] I. F. Herbut, V. Juricic, and O. Vafek, Phys. Rev. Lett. 100, 046403 (2008).

[15] O. Vafek and M. J. Case, Phys. Rev. B 77, 033410 (2008).

[16] E. Barnes, E. H. Hwang, R. E. Throckmorton, and S. Das Sarma, Phys. Rev. B 89, 235431 (2014).

[17] C. Bauer, A. Rückriegel, A. Sharma, and P. Kopietz, Phys. Rev. B 92, 121409 (2015).

[18] A. Sharma and P. Kopietz, Phys. Rev. B 93, 235425 (2016).

[19] S. H. Abedinpour, G. Vignale, A. Principi, M. Polini, W.-K. Tse, and A.H. MacDonald, Phys. Rev. B 84, 045429 (2011).

[20] E. G. Mishchenko, Europhys. Lett. 83, 17005 (2008).

[21] D. E. Sheehy and J. Schmalian, Phys. Rev. B 80, 193411 (2009).

[22] V. Juricic, O. Vafek, and I. F. Herbut, Phys. Rev. B 82, 235402 (2010).

[23] I. Sodemann and M. M. Fogler, Phys. Rev. B 86, 115408 (2012).

[24] B. Rosenstein, M. Lewkowicz, and T. Maniv, Phys. Rev. Lett. 110, 066602 (2013).

[25] S. Teber and A. V. Kotikov, Europhys. Lett. 107, 57001 (2014).

[26] J. M. Link, P. P. Orth, D. E. Sheehy, and J. Schmalian, Phys. Rev. B 93, 235447 (2016).

[27] D. L. Boyda, V. V. Braguta, M. I. Katsnelson, and M. V. Ulybyshev, Phys. Rev. B 94, 085421 (2016).
[28] I. Ozfidan, M. Korkusinski, and P. Hawrylak, Phys. Rev. B 91, 115314 (2015).

[29] M. Trushin and J. Schliemann, Phys. Rev. Lett. 107, 156801 (2011).

[30] M. Trushin and J. Schliemann, New J. Phys. 14, 095005 (2012).

[31] J. Jung and A. H. MacDonald, Phys. Rev. B 84, 085446 (2011).

[32] A. Giuliani, V. Mastropietro, and M. Porta, Ann. Phys. (Amsterdam) 327, 461 (2012).

[33] N. Y. Astrakhantsev, V. V. Braguta, and M. I. Katsnelson, Phys. Rev. B 92, 245105 (2015).

[34] D. N. Basov, R. D. Averitt, D. van der Marel, M. Dressel, and K. Haule, Rev. Mod. Phys. 83, 471 (2011).

[35] L. S. Levitov, A. V. Shtyk, and M. V. Feigelman, Phys. Rev. B 88, 235403 (2013).

[36] L. Wu, W.-K. Tse, M. Brahlek, C. M. Morris, R. V. Aguilar, N. Koirala, S. Oh, and N. P. Armitage, Phys. Rev. Lett. 115, 217602 (2015).

[37] K. W. Post, B. C. Chapler, M. K. Liu, J. S. Wu, H. T. Stinson, M. D. Goldflam, A. R. Richardella, J. S. Lee, A. A. Reijnders, K. S. Burch, M. M. Fogler, N. Samarth, and D. N. Basov, Phys. Rev. Lett. 115, 116804 (2015).

[38] T. Stauber, J. Phys. Condens. Matter 26, 123201 (2014).

[39] See Supplemental Material at http://link.aps.org/ supplemental/10.1103/PhysRevLett.118.266801, which also includes Refs. [40-48].

[40] G. Giuliani and G. Vignale, Quantum Theory of the Electron Liquid (Cambridge Cambridge University Press, Cambridge, England, 2005).

[41] T. Stauber, J. Schliemann, and N. M. R. Peres, Phys. Rev. B 81, 085409 (2010).

[42] T. Stauber, Phys. Rev. B 82, 201404 (2010).

[43] T. O. Wehling, E. Şaşıŏ̆lu, C. Friedrich, A. I. Lichtenstein, M. I. Katsnelson, and S. Blügel, Phys. Rev. Lett. 106, 236805 (2011).

[44] M. V. Ulybyshev, P. V. Buividovich, M. I. Katsnelson, and M. I. Polikarpov, Phys. Rev. Lett. 111, 056801 (2013).

[45] P. V. Buividovich and M. I. Polikarpov, Phys. Rev. B 86, 245117 (2012).

[46] D. Smith and L. von Smekal, Phys. Rev. B 89, 195429 (2014).

[47] B. B. Brandt, A. Francis, H. B. Meyer, and D. Robaina, Phys. Rev. D 92, 094510 (2015).

[48] P. Buividovich, D. Smith, M. Ulybyshev, and L. von Smekal, Proceedings of the 34th International Symposium on Lattice Field Theory (Lattice 2016): Southampton, UK, July 24-30, 2016, Proc. Sci., LATTICE2016 (2016) 244 [arXiv:1610.09855].

[49] J. E. Drut and T. A. Lähde, Phys. Rev. Lett. 102, 026802 (2009).

[50] E. C. Marino, L. O. Nascimento, V.S. Alves, and C. M. Smith, Phys. Rev. X 5, 011040 (2015).

[51] C. L. Kane and E. J. Mele, Phys. Rev. Lett. 93, 197402 (2004).

[52] S. Reich, J. Maultzsch, C. Thomsen, and P. Ordejón, Phys. Rev. B 66, 035412 (2002).

[53] T. Stauber and N. M. R. Peres, J. Phys. Condens. Matter 20, 055002 (2008).

[54] N. M. R. Peres and T. Stauber, Int. J. Mod. Phys. B 22, 2529 (2008). 